\title{
Signal-to-Noise Ratio Improvement based on the Discrete Wavelet Transform in Ultrasonic Defectoscopy
}

\author{
V. Matz, M. Kreidl, R. Šmíd
}

In ultrasonic testing it is very important to recognize the fault echoes buried in a noisy signal. The fault echo characterizes a flaw in the material. An important requirement on ultrasonic signal filtering is zero-time shift, because the position of ultrasonic echoes is essential. This requirement is accomplished using the discrete wavelet transform (DWT), which is used for reducing the signal-to-noise ratio. This paper evaluates the quality of filtering using the discrete wavelet transform. Additional computer simulations of the proposed algorithms are presented.

Keywords: ultrasonic testing, discrete wavelet transform, de-noising algorithms.

\section{Introduction}

Ultrasonic non-destructive testing is used for detecting flaws in materials. Ultrasound uses the transmission of high-frequency sound waves in a material to detect a discontinuity or to locate changes in material properties. The most commonly used ultrasonic testing technique is a pulse echo, where sound is introduced into a test object and the reflections (echoes) are returned to a receiver from internal imperfections or from the geometrical surfaces of a part. The highest signal-to-noise ratio (SNR) provides the optimum frequency of an acoustic wave appropriate for detecting specific discontinuity. There are several sources of noise that can hide a fault. A common source of noise is electronic circuitry, which is used for processing the ultrasonic signal, and scattering at the inhomogeneities in the structure of a grainy material. The amplitude of the fault echoes can be smaller than the amplitude of the noise, and the noise can totally mask echoes characterizing faults. This case is undesirable, because we cannot correctly identify flaws in the material. The most frequent usage of ultrasonic testing is for weld inspection. In welds there is big probability of cracking. The places where the flaws are have to be uniquely determined. For this determination we have to use a method for reducing the ultrasonic signal noise. The best method for reducing noise which ensures zero-time shifts of ultrasonic echoes is the discrete wavelet transform (DWT) [1].

\section{Filtering method based on the discrete wavelet transform}

The wavelet transform is a multiresolution analysis technique that can be used to obtain the time-frequency representation of the ultrasonic signal. The continuous wavelet transform (CWT) is computed by changing the scale of the analysis window, shifting the window in time, multiplying by the signal, and integrating over all times. The continuous wavelet transform is defined by:

$$
C W T_{\tau}^{\psi}(\tau, s)=\frac{1}{\sqrt{|s|}} \int x(t) \Psi *\left(\frac{t-\tau}{s}\right) \mathrm{d} t,
$$

where $x(t) \quad$ is the input signal, $t \quad$ is the translation,

$\mathrm{s} \quad$ is the scale and

$\Psi(t) \quad$ is the transforming function called mother wavelet.

The mother wavelet is given by:

$$
\Psi_{\tau, s}=\frac{1}{\sqrt{s}} \Psi\left(\frac{t-\tau}{s}\right) .
$$

DWT coefficients are usually sampled from the CWT on a dyadic grid, choosing parameters of translation $\tau=n \cdot 2^{m}$ and scale $s=2^{m}$, it is possible to defined mother wavelet in DWT as:

$$
\Psi_{m, n}(t)=\frac{1}{\sqrt{2^{m}}} \Psi\left(\frac{t-n 2^{m}}{2^{m}}\right) .
$$

DWT $[2,3]$ analyzes the signal by decomposing it into its coarse and detail information, which is accomplished by using successive high-pass and low-pass filtering operations, on the basis of the following equations:

$$
\begin{aligned}
& y_{\text {high }}(k)=\sum_{n} x(n) \cdot g(2 k-n), \\
& y_{\text {low }}(k)=\sum_{n} x(n) \cdot h(2 k-n),
\end{aligned}
$$

where $y_{\text {high }}(k)$ and $y_{\text {low }}(k)$ are the outputs of the high-pass and low-pass filters with impulse response $g$ and $h$, respectively, after subsampling by 2 . This procedure is repeated for further decomposition of the low-pass filtered signals.

Starting from the approximation and detailed coefficients the inverse discrete wavelet reconstructs signal, inverting the decomposition step by inserting zeros and convolving the results with the reconstruction filters.

The discrete wavelet transform $[4,5]$ can be used as an efficient filtering method for families of signals that have a few nonzero wavelet coefficients for a given wavelet family. This is fulfilled for most ultrasonic signals. The standard filtering (also called de-noising) procedure affects the signal in both frequency and amplitude, and involves three steps. The basic version of the procedure consists of: 
a) decomposition of the signal using DWT into $N$ levels using bandpass filtering and decimation to obtain the approximation and detailed coefficients,

b) thresholding of detailed coefficients (see Fig. 1),

c) reconstruction of the signal from detailed and approximation coefficients using the inverse transform (IDWT).

For decomposition of the signal it is very important to choose a suitable mother wavelet. The shape of the mother wavelet has to be very similar to the ultrasonic echo. It has to fulfill the following properties: symmetry, orthogonality and feasibility for DWT. A group of mother wavelets was tested: Haar's wavelet, the discrete Meyer wavelet, Daubechie's wavelet and Coiflet's wavelet. The best results were obtained with the discrete Meyer wavelet. In the following study, only this mother wavelet was used.
In the proposed procedure, local thresholding of detailed coefficients was used $[6,7]$. We computed the threshold at each level of decomposition from the detailed coefficients, and this value was used for thresholding in the same level. We evaluated common thresholding methods implemented in the Matlab Wavelet toolbox [8] (rigsure, sqtwolog, heursure, minimaxi) and due to the unsatisfactory results we proposed a new method based on standard deviation. The local threshold at every level of decomposition is given by

$$
\text { Threshold }=k \cdot \sqrt{\frac{1}{N-1} \cdot \sum_{i=1}^{N}\left(\boldsymbol{D} \boldsymbol{c}_{i}-\overline{\boldsymbol{D}} \boldsymbol{c}\right)^{2}}
$$

where $k$ is the coefficient related to the crest factor of the filtered signal,

$D \boldsymbol{c}$ is a vector of detailed coefficients at each level,

$N$ is the length of each set of detailed coefficients.

Input data

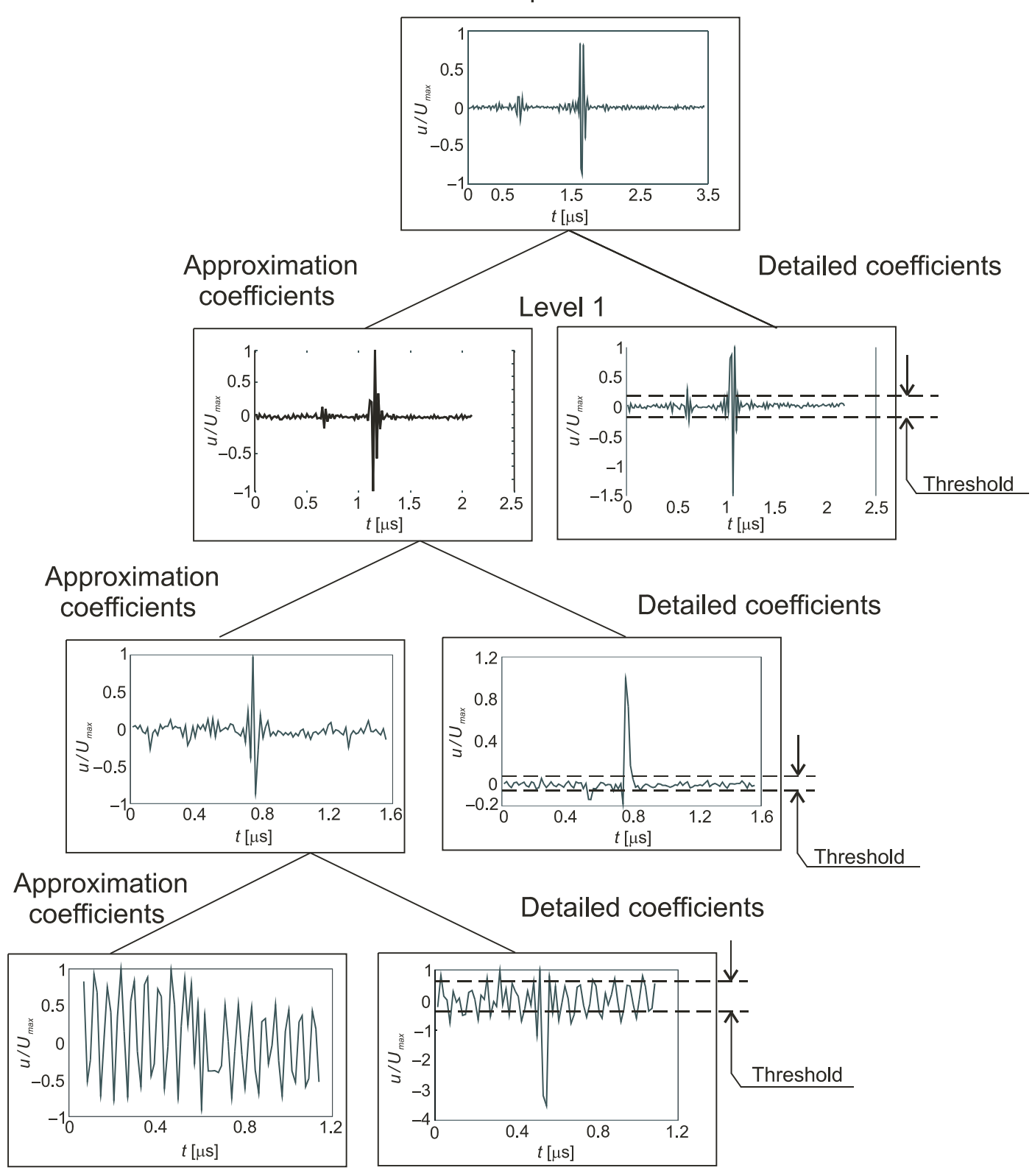

Fig. 1: Decomposition and tresholding 


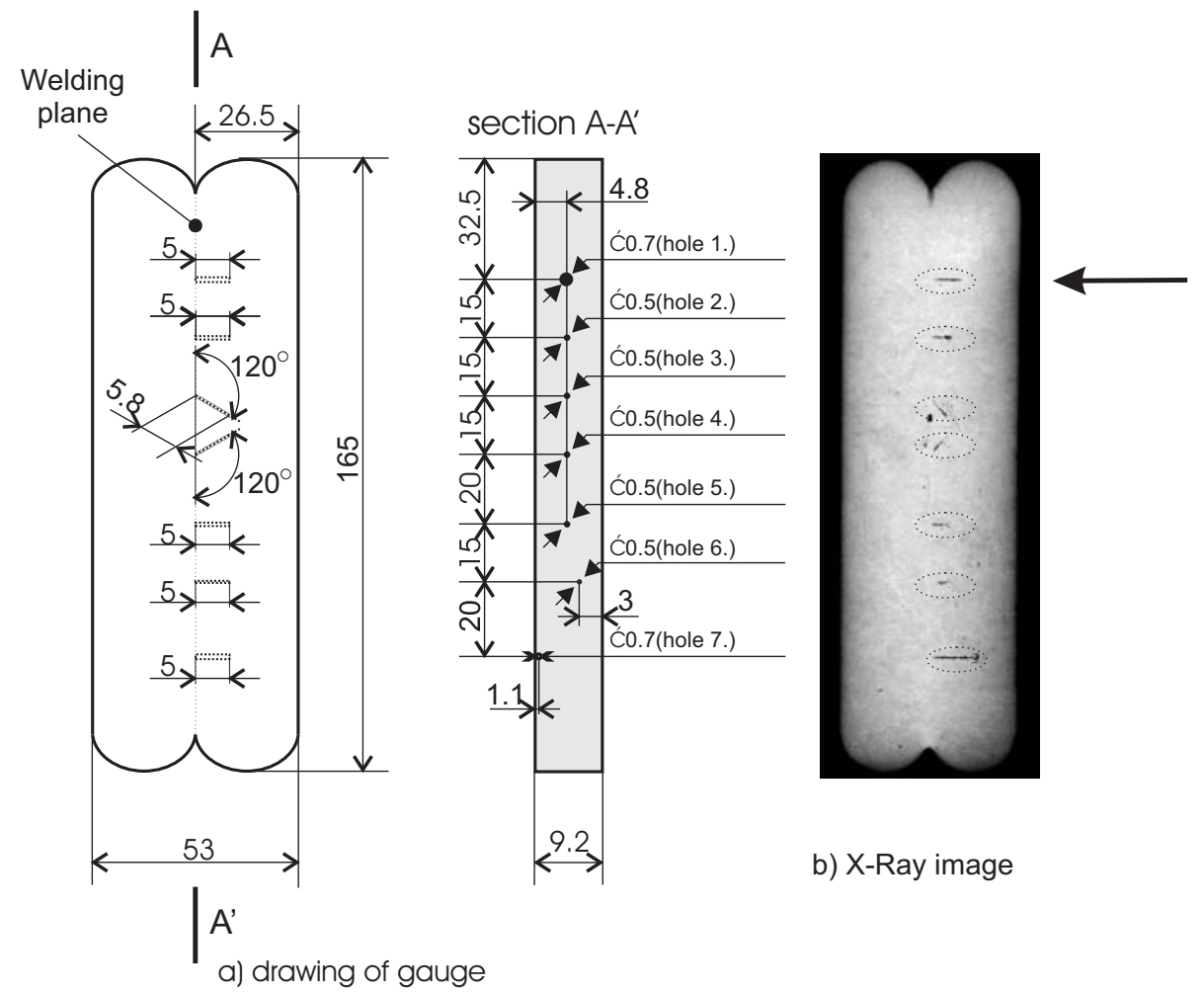

Fig. 2: Material used for constructing airplane engines

The main idea of DWT filtering involves replacing small wavelet coefficients by zero, and keeping the coefficients with an absolute value above the threshold. This type of thresholding is called hard thresholding [7], and is used in our study.

To evaluate the noise reduction we used the signal measured on grainy material used for constructing airplane engines (see Fig. 2). The ultrasonic signal from this material is very noisy. The noise is partially caused by scattering at the grains in the structure of the materials.

The arrow in Fig. 2 shows the place where the measurement was conducted. Fig. 3 shows the raw signal in the place

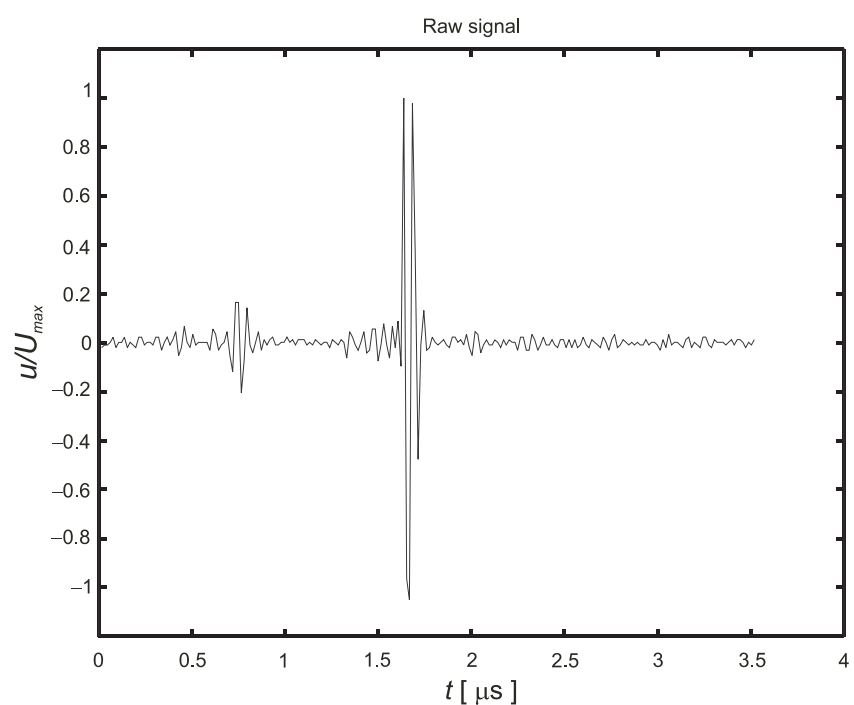

where crack No.1 was located. This crack was artificially created.

Fig. 3 shows noise reduction, but the sources of this noise are not fully known. To determine the filtering quality a standard K1 calibration gauge was used. The gauge is made of homogeneous material so the noise can be estimated and fully described. We made a measurement of the ultrasonic signal which took into consideration only back-wall echo. For a comparison with the previous signal, we composed the artificial fault echo from a properly scaled back-wall echo. In our study the amplitude of the fault echo from $5 \%$ to $100 \%$ was changed. To evaluate the filtration quality we used the signal-to-noise improvement ratio is used:

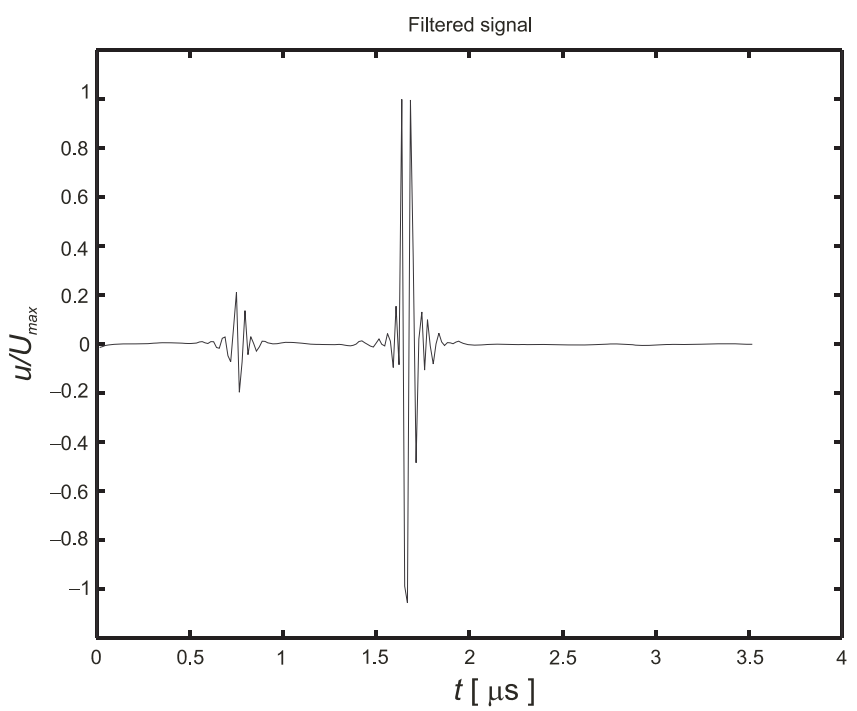

Fig. 3: Filtering of an ultrasonic signal using DWT with the threshold based on standard deviation 

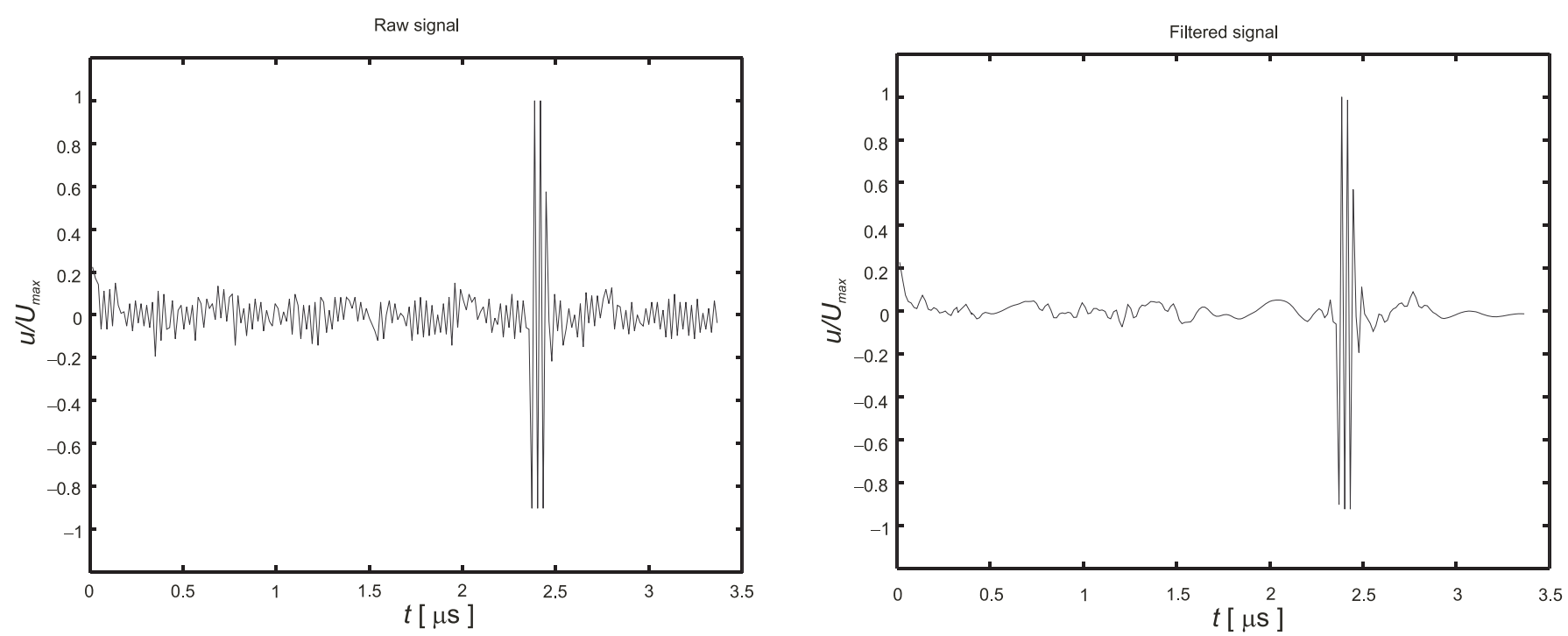

Fig. 4: Filtering of an ultrasonic signal without fault echo
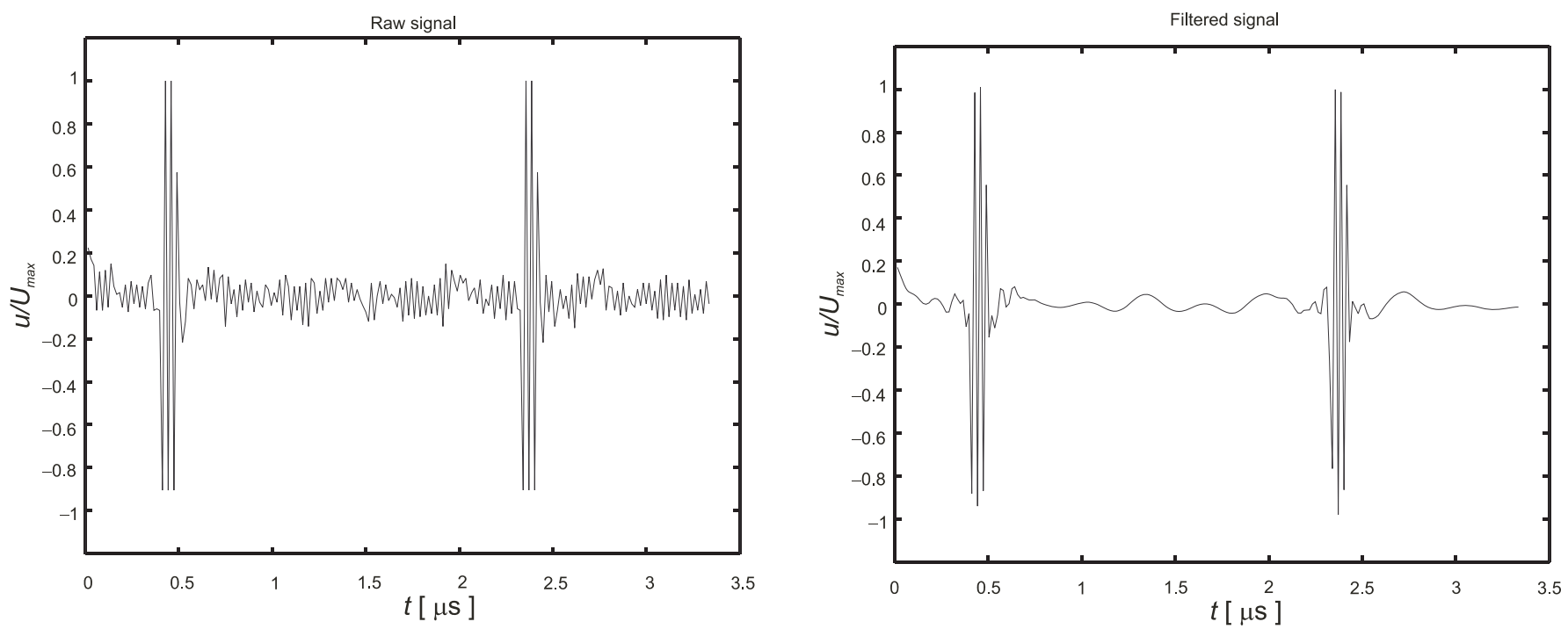

Fig. 5: Filtering of an ultrasonic signal with $100 \%$ fault echo
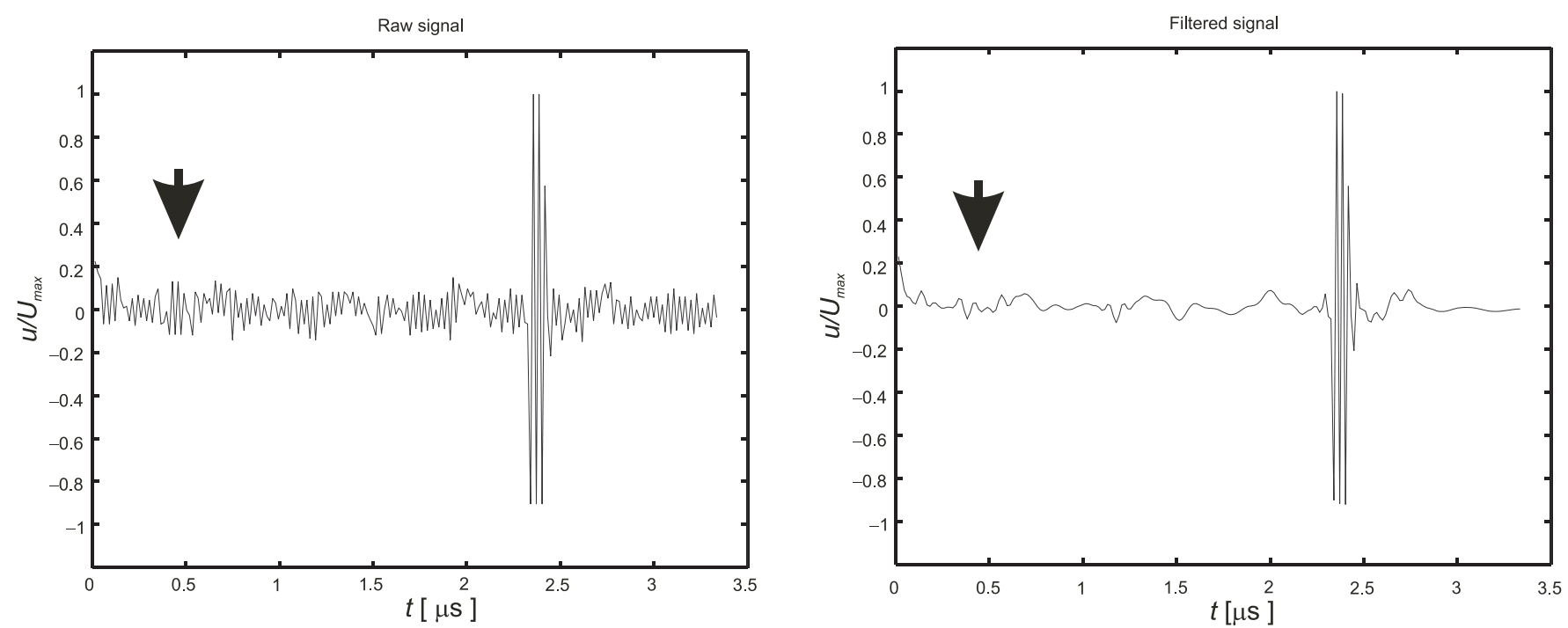

Fig. 6: Filtering of an ultrasonic signal - fault echo with amplitude $132.6 \%$ of back-wall echo 


$$
F N R=20 \log \left(\frac{F_{\text {ef }}}{N_{\text {ef }}}\right)[\mathrm{dB}],
$$

where $N_{\text {ef }}$ is the root mean square value of the noisy part of the raw signal,

$F_{\text {ef }}$ is the root mean square value of an adequate part of the filtered signal.

\section{Experimental results}

For filtering the ultrasonic signal measured on the K1 calibration gauge we used the same filtering technique based on DWT as in the previous case. The following figures show the filtering signal without fault echo (see Fig. 4) and with fault echo (see Fig. 5), which has the same amplitude (100\%) as the back-wall echo.

Table 1: Noise reduction for different amplitudes of fault echo

\begin{tabular}{|c|c|c|c|}
\hline Amplitude [\%] & $F N R[\mathrm{~dB}]$ & Amplitude [\%] & $F N R[\mathrm{~dB}]$ \\
\hline 51 & 17.7 & 357 & 18.4 \\
\hline 102 & 17.7 & 408 & 18.4 \\
\hline 112.2 & 17.7 & 459 & 18.3 \\
\hline 122.4 & 17.7 & 510 & 18.3 \\
\hline 132.6 & 17.7 & 561 & 18.3 \\
\hline 142.8 & 17.7 & 612 & 18.3 \\
\hline 153 & 17.7 & 663 & 18.2 \\
\hline 163.1 & 17.7 & 714 & 18.2 \\
\hline 173.4 & 17.7 & 765 & 19.0 \\
\hline 183.5 & 17.7 & 816 & 19.0 \\
\hline 194 & 17.7 & 867 & 18.9 \\
\hline 204 & 17.6 & 918 & 19.7 \\
\hline 255 & 17.6 & 968 & 19.7 \\
\hline 306 & 17.9 & 1020 & 19.6 \\
\hline
\end{tabular}

A value of $1020 \%$ of effective noise value corresponds to $100 \%$ of back-wall echo. The results presented in Table 1 show that the noise reduction value varies from 17 to $20 \mathrm{~dB}$.

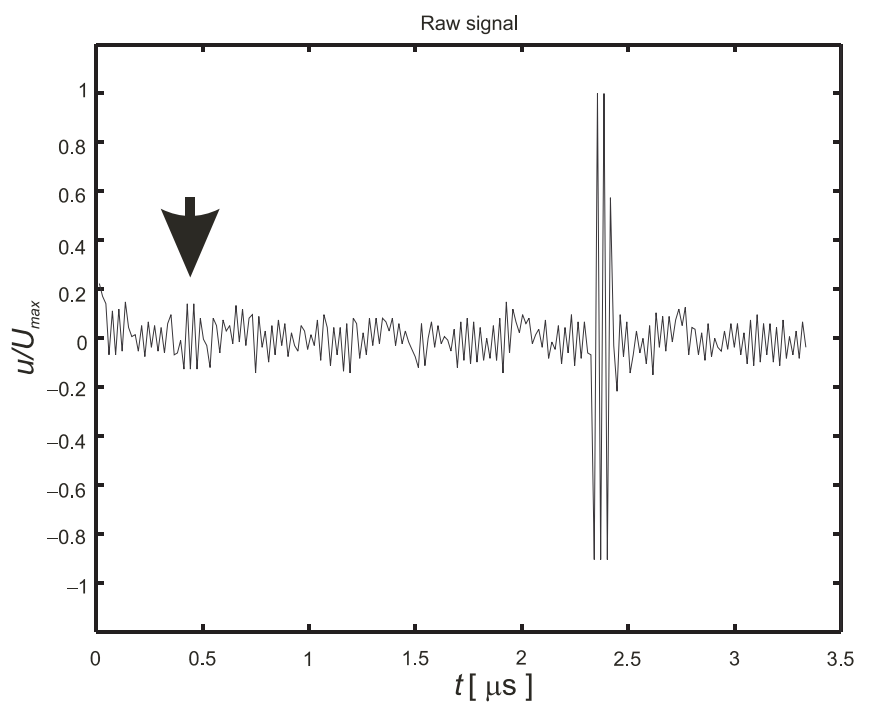

For relative amplitude $132.6 \%$ no fault echo can be identified, but for relative amplitude higher than $132.6 \%$ the fault echo can be recognized. The results for relative amplitudes of $132.6 \%$ and $142.8 \%$ are depicted in Fig. 6 and Fig. 7. The arrows indicate the fault echo that can be hidden by noise.

The proposed algorithm based on filtering using the discrete wavelet transform was tested on data measured on two materials: a K1 calibration gauge and a construction material used in airplane engines. A simulated fault was created which artificially reduced the back-wall echo and was inserted in the raw signal. The results of the measurements are shown in Fig. 6 and Fig. 7.

\section{Conclusion}

This paper describes a method for filtering an ultrasonic signal using the discrete wavelet transform. For thresholding we used a novel thresholding technique based on the standard deviation of coefficients of DWT. This method provides the best result for filtering of simulated and real ultrasonic signals. The noise reduction for a signal without fault echo is $18.56 \mathrm{~dB}$. For signals with a simulated fault echo the noise reduction ratio was from $17.65 \mathrm{~dB}$ to $19.72 \mathrm{~dB}$. We also investigated improvements in sensitivity of fault detection. Our method allows identification of faults with relative amplitude higher than $132.6 \%$ of the effective noise value.

\section{Acknowledgment}

This research work has received support from research program No. MSM210000015 "Research of New Methods for Physical Quantities Measurement and Their Application in Instrumentation" of the Czech Technical University in Prague (sponsored by the Ministry of Education, Youth and Sports of the Czech Republic ).

\section{References}

[1] Šmíd R., Matz V., Kreidl M.: "Ultrasonic signal filtering.” Defektoskopie 2003. Praha: Česká společnost pro nedestruktivní testování, 2003, ISBN 80-214-2475-3, p. 259-262.

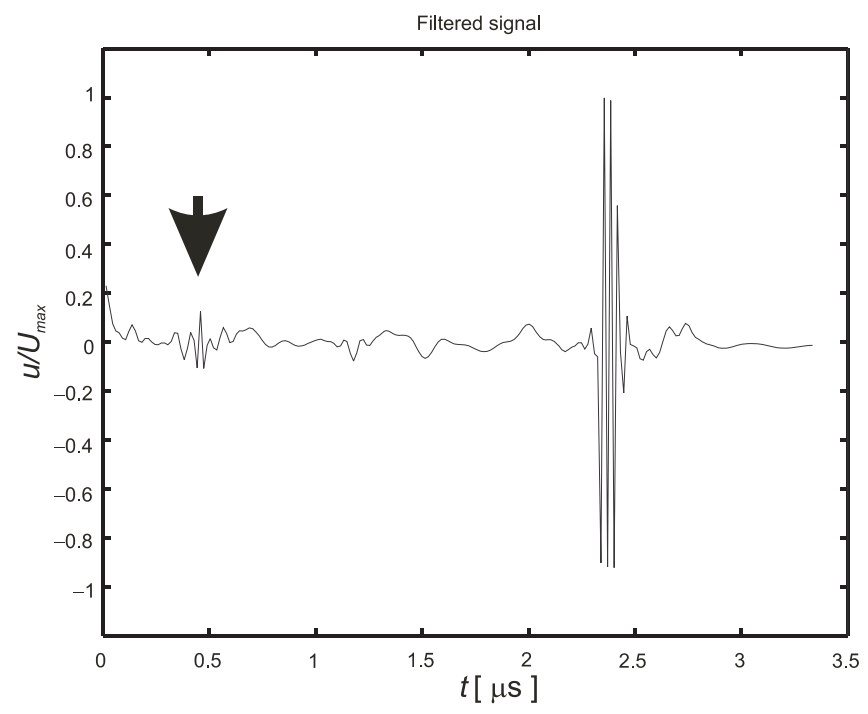

Fig. 7: Filtering of an ultrasonic signal - fault echo with relative amplitude of $142.8 \%$ of back-wall echo 
[2] Edwards T.: Discrete wavelet transforms: Theory and implementation. Technical report, Stanford University, September 1992.

[3] Louis A. K., Maaß P., Rieder A.: Wavelets: Theory and Applications. John Wiley and Sons Ltd., England, 1997.

[4] Mallat S.: A Wavelet Tour of Signal Processing. Academic Press, 1999.

[5] Kreidl M. et al.: Diagnostic Systems (in Czech). Czech Technical University in Prague, Prague, 2001. 352 p. ISBN 80-01-02349-4.

[6] Polikar R. et al.: "Frequency Invariant Classification of Ultrasonic Weld Inspection Signal.” IEEE Trans. On Ultrasonic, Ferro. And Freq.Contr., Vol. 45, No. 3, May, 1998.

[7] Polikar R.: The engineer's ultimate guide to wavelet analysis. Iowa State University of Science and Technology, 1999, http://users.rowan.edu/ polikar/WAVELETS/.

[8] Misiti M., Misiti Y., Oppenheim G., Poggi J-M.: Wavelet Toolbox For Use with MATLAB, User's Guide, version 2. The MathWorks, Inc., 2002.

Ing. Václav Matz

phone: +420224352346

e-mail:vmatz@email.cz

Doc. Ing. Marcel Kreidl, CSc.

phone: +420224352117

e-mail: kreidl@feld.cvut.cz

Ing. Radislav Šmíd, Ph.D.

phone: +420224352131

e-mail: smid@feld.cvut.cz

Department of Measurement

Czech Technical University in Prague

Faculty of Electrical Engineering

Technická 2

16627 Praha 6, Czech Republic 